\title{
Radio spectra generated during coalescence processes of plasmoids in a flare current sheet
}

\author{
M. Karlický1 ${ }^{1}$ M. Bárta ${ }^{1,3}$, and J. Rybák ${ }^{2}$ \\ 1 Astronomical Institute of the Academy of Sciences of the Czech Republic, 25165 Ondřejov, Czech Republic \\ e-mail: karlicky@asu.cas.cz \\ 2 Astronomical Institute, Slovak Academy of Sciences, 05960 Tatranská Lomnica, Slovak Republic \\ 3 Max Planck Institute for Solar System Research, 37191 Katlenburg-Lindau, Germany
}

Received 26 October 2009 / Accepted 23 January 2010

ABSTRACT

\begin{abstract}
Aims. Motivated by observations of the drifting pulsating structures (DPSs) in solar radio spectra, we study the electromagnetic (radio) emission generated during tearing and coalescence processes in a flare current sheet.

Methods. For numerical simulations, we used a 2.5-D particle-in-cell electromagnetic relativistic code. Numerical data were analyzed by the wavelet methods.

Results. It is found that the electromagnetic emission is generated during a coalescence of plasmoids, and it has a quasi-periodic character. Detailed analysis reveals that the electromagnetic emission is produced around the interacting plasmoids just before their coalescence into a larger one. The period in variations of electromagnetic emission corresponds to that of magnetic field at the same region. Reflections of the electromagnetic waves between interacting plasmoids are recognized. The computed and observed periodicities are discussed. The similarity of the DPSs with some radio bursts observed during star flares indicates a broader applicability for this model.
\end{abstract}

Key words. Sun: flares - Sun: radio radiation

\section{Introduction}

As shown in the papers by Kliem et al. (2000), Khan et al. (2002), and Karlický (2004), there is a close relationship between the drifting pulsating structures (DPSs) observed on radio waves in the decimetric range and the plasmoid ejection observed in X-rays (Ohyama \& Shibata 1998). Kliem et al. (2000) propose that the DPSs are generated during a bursting regime of the magnetic field reconnection when plasmoids (magnetic islands) are formed. It has been suggested that the plasmoids mutually interact and during this process electrons are accelerated. These electrons are trapped in the plasmoid and generate DPS through the plasma emission mechanism on the plasma frequency corresponding to the plasma density inside the plasmoid.

The global frequency drift of DPS is explained by a motion of the plasmoid in the density gradient of the solar atmosphere. A divergence in magnetic field lines in the upward direction in the solar atmosphere means that the upward motion of the plasmoid is preferred (Bárta et al. 2008b), so most of DPSs drift towards lower frequencies. Nevertheless, there are also the cases when the plasmoids move downwards and the associated DPSs drift to higher frequencies. The plasma density inside the plasmoid differs from that in the surrounding solar atmosphere, therefore this difference needs to be considered in the height estimation of the DPS source in the solar atmosphere from its DPS spectrum (for more details, see Bárta et al. 2008a). An example of the DPS observed at the beginning of the May 21, 2002 flare is shown in Fig. 1. As seen here this radio burst is characterized by a quasi-periodic series of short-lasting pulses (some of them with fast frequency drifts), which as a whole are narrowbanded and slowly drifting. The DPSs are usually observed in the frequency range $0.6-2 \mathrm{GHz}$, which corresponds to the plasma densities in the radio sources $n_{\mathrm{e}}=4.4 \times 10^{9}-4.9 \times 10^{10} \mathrm{~cm}^{-3}$. The global drift of DPSs is typically in the range $-20-20 \mathrm{MHz} \mathrm{s}^{-1}$ (Bárta et al. 2008a).

The concept of the generation of DPS was numerically analyzed in the paper by Karlický \& Bárta (2007), where it was shown how the electrons are accelerated during a coalescence of two plasmoids and how the electrons are trapped in the plasmoids. A presence of electromagnetic waves was shown by the dispersion diagrams.

In the present paper we continue in our previous studies, we present details of generation of the electromagnetic (radio) waves during the coalescence of plasmoids, we compute their spectra in the same form as the standard radio spectra, and we study periodicities in these processes. We want to answer the question if this electromagnetic (radio) emission has a quasiperiodic character as typical of DPSs (Fig. 1).

The layout of the paper is as follows. In Sect. 2 we present the model, then the results of the simulations are shown in Sect. 3. Discussions and conclusions are in Sects. 4 and 5.

\section{Model}

To simulate the processes under study, we use a 2.5-D (2D3V 2 spatial and 3 velocity components) fully relativistic electromagnetic particle-in-cell (PIC) code (Saito \& Sakai 2004). The system size is $L_{x}=4000 \Delta$ and $L_{y}=600 \Delta$, where $\Delta(=1)$ is a grid size. 


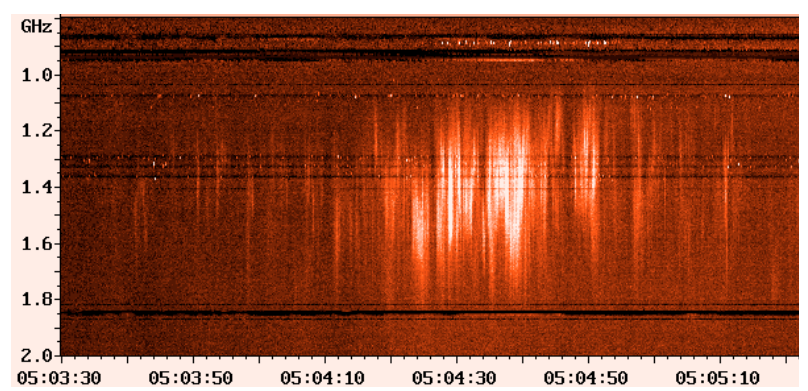

Fig. 1. The $0.8-2.0 \mathrm{GHz}$ radio spectrum showing the drifting pulsating structure observed by the Ondřejov radiospectrograph (Jiřička et al. 1993) at 05:03:30 - 05:05:26 UT on May 21, 2002.

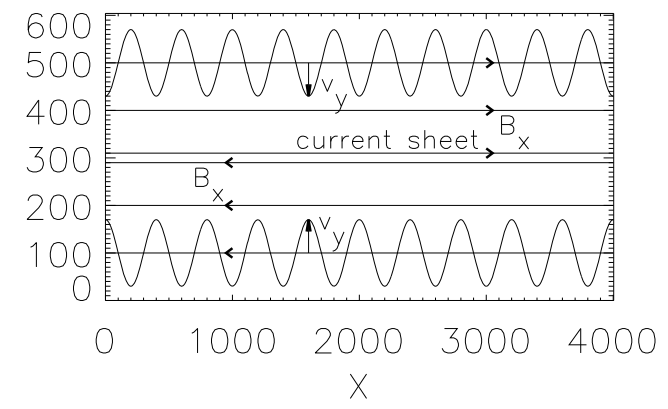

Fig. 2. Initial conditions of the simulation. Horizontal lines with arrows are magnetic field lines forming a current sheet. Values of the initial plasma velocities $v_{y}$ on both sides of the current sheet are expressed by the cosine-like lines. Free boundary conditions are considered.

The current sheet is initiated along the line $y=y_{0}=300 \Delta$, see the initial setup in Fig. 2. The half-width of the current sheet is $L=10 \Delta$. The initial magnetic field is

$$
\begin{aligned}
\boldsymbol{B} & \equiv\left(B_{x}, B_{y}, B_{z}\right), \\
B_{x} & =-B_{0} \text { for } y<y_{0}-L, \\
B_{x} & =\left(y-y_{0}\right) B_{0} / L \text { for } y_{0}-L \leq y \leq y_{0}+L, \\
B_{x} & =B_{0} \text { for } y>y_{0}+L, \\
B_{y} & =0, B_{z}=0 .
\end{aligned}
$$

In this first study, we have considered the neutral current sheet, i.e. with no guiding magnetic field.

The electron-proton plasma with the proton-electron mass ratio $m_{\mathrm{p}} / m_{\mathrm{e}}=16$ is unrealistic, but taken to shorten proton skin depth and computations. Nevertheless, the electron mass is low enough to separate the dynamics of electrons and protons well. In each numerical cell located outside of the current sheet, we initiated $n_{0}=60$ electrons and $n_{0}=60$ protons. In the current sheets the initial number density was enhanced just to keep the pressure equilibrium. The electron thermal velocity was taken to be the same in the whole numerical box as $v_{\mathrm{Te}}=0.1 c$, where $c$ is the speed of light. The corresponding electron temperature is $T=59.5 \mathrm{MK}$. The temperature of protons was chosen the same as electrons.

The appropriate electric current density was initiated in the current sheet. The Debye length corresponds to the grid size $\Delta$. The plasma beta parameter and the ratio of the electroncyclotron and electron-plasma frequencies in the region outside the current sheet were chosen as $\beta=0.2$ and $\omega_{\mathrm{ce}} / \omega_{\mathrm{pe}}=0.32$. The Alfven speed is $v_{\mathrm{A}}=0.078 c$. Free boundary conditions we considered.

We tried to choose these parameters as close to the real flare conditions as possible. We considered the low-beta plasma and the radio emission frequency of the DPS close to the electron plasma frequency as assumed in most models of solar dm-radio bursts (the ratio $\omega_{\text {ce }} / \omega_{\text {pe }}$ is then less than 1 ), and see the models of the magnetic field and plasma densities in the low corona e.g., in Aschwanden (2004). But because of the PIC model limits and shortening of computing times we used temperatures that are higher than in preflare corona. From the physical point of view, we can assume the higher temperatures as a result of the heating in the pre-flare heating phase. But the plasma temperature and the proton-electron mass ratio have weak or negligible effects on the electron plasma frequency, which is the main parameter in this paper.

We wanted to study several coalescence processes among several plasmoids, but in our current-sheet system without any further perturbations, the tearing mode instability only produces two plasmoids with sizes of a half of the length of the current sheet (see Karlický \& Bárta 2007). Therefore, in the initial state, we triggered a formation of 10 plasmoids by a cosine-type plasma flow counter-streaming from the opposite sides of the current sheet as (Fig. 2)

$$
\begin{aligned}
v & \equiv\left(v_{x}, v_{y}, v_{z}\right), \\
v_{x} & =0, \\
v_{y} & =-A \cos (2 \pi 10 x / L) \text { for } y>y_{0}, \\
v_{y} & =A \cos (2 \pi 10 x / L) \text { for } y<y_{0}, \\
v_{z} & =0,
\end{aligned}
$$

where $v$ is the plasma flow velocity, $L$ the system length, and $A$ was chosen as $A=0.01 \mathrm{c}$.

The wavelet transform was used to analyze the E and B signals in the particular parts of the time-space domain. The signals were transformed using the Morlet transform specifying the parameter relating the sine wave and the Gaussian window $\omega=6$ (Torrence \& Compo 1998) in the selected intervals of time and plasma frequency. The cone of influence was determined in regions where edge effects become important due to finite length of the signals. The thick solid black lines in the plots of the wavelet power spectra stem from the confidence level relative to red noise.

All computations were performed on the parallel computer OCAS (Ondřejov Cluster for Astrophysical Simulations), see http: //wave.asu.cas.cz/ocas.

\section{Results}

As a result of the initial plasma flow, 10 small plasmoids were formed in the very early stage of evolution, see Fig. 3A at 79.6 plasma periods $\left(\omega_{\mathrm{pe}} t=500\right)$. (The tearing-mode instability and free boundary conditions means that the small plasmoids at both boundaries are not symmetric.) Then these plasmoids coalesce into larger and larger plasmoids (Fig. 3). At each time step, we recorded values of the full vectors of the magnetic and electric fields $(\boldsymbol{B}$ and $\boldsymbol{E})$ along the line $y=300 \Delta$, i.e., just along the line where the plasmoids interact. Figure 4 shows a time evolution of the absolute value of the magnetic field along this line. This figure describes how the small plasmoids coalesce step-by-step into larger ones, see channels, delimited by thicker lines, which mark regions with very low values of the magnetic field in plasmoids (O-type islands).

Then in different locations along the $y=300 \Delta$ line, we started to analyze the $E_{z}$ component of the electric field (the component perpendicular to the computational plane), which corresponds to the electromagnetic waves (radio for DPSs) in 

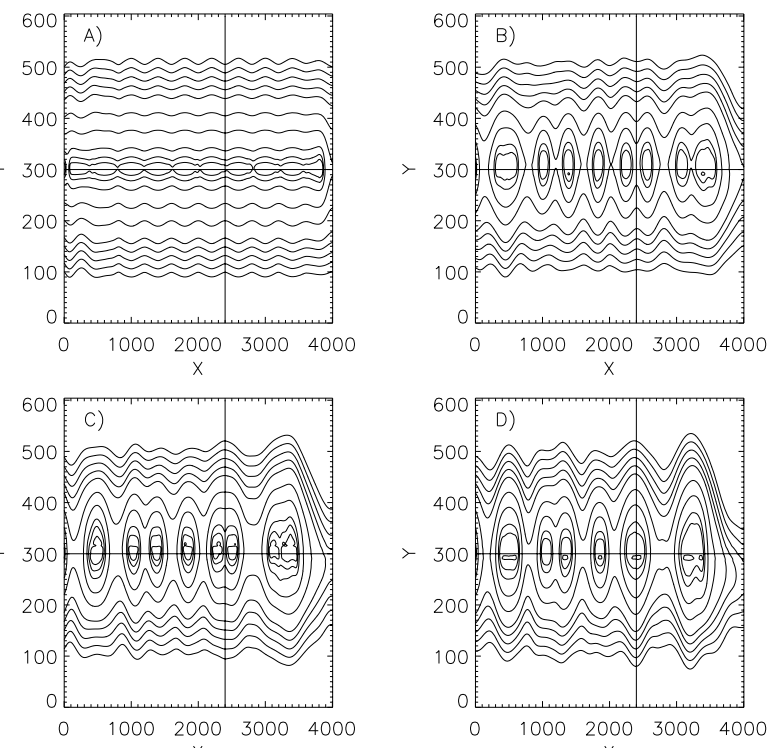

Fig. 3. Magnetic field lines showing tearing and coalescence processes in the neutral current sheet at four different times: A) at 79.6 plasma periods, B) at 198.9 plasma periods, C) at 238.7 plasma periods, and D) at 278.5 plasma periods. The horizontal line denotes the line $y=300 \Delta$, where E) and B) were recorded. The vertical line shows the location between two interacting plasmoids, where processes were studied $(x=$ 2400A).

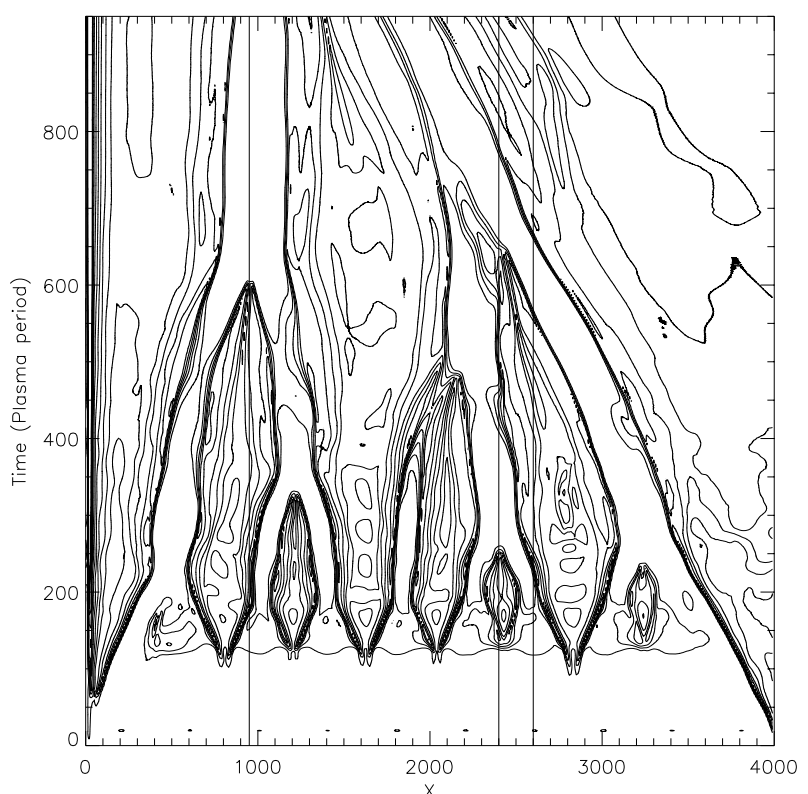

Fig. 4. The contours of the absolute value of the magnetic field along the line $y=300 \Delta$ (see Fig. 3) as they reveal the time dependence of the kinematics of plasmoids. The vertical lines show the locations important in our study ( $x=950,2400$, and 2600 $\Delta$ ).

this system. It was also checked by the computation of the dispersion diagrams as by Karlický \& Bárta (2007). We found that the electromagnetic emission at frequencies close to the local plasma frequency is generated at the locations between coalescing plasmoids, as shown in the wavelet spectrum taken at the location $x=2400 \Delta$ (see Figs. 3 and 4), in the time interval 120-270 plasma periods (Fig. 5). As seen in Fig. 5, which has a form of the standard radio spectrum, the emission is characterized by quasi-periodic pulsations in the frequency range of $0.75-2.0$ initial plasma frequency. Furthermore, it was

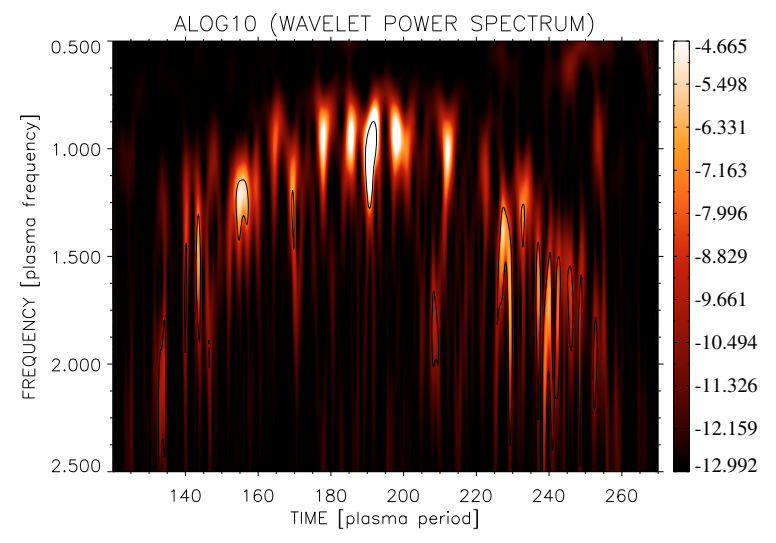

Fig. 5. The wavelet spectrum of the $E_{\mathrm{z}}$ component of the electric field at the location $x=2400 \Delta$ and $y=300 \Delta$ at times $120-270$ plasma periods.

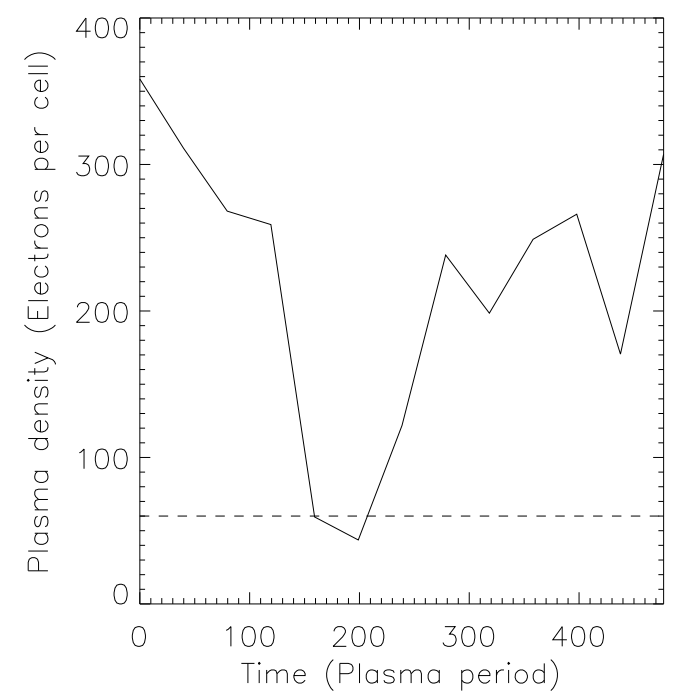

Fig. 6. Time evolution of the plasma density at the location $x=2400 \Delta$ and $y=300 \Delta$. The horizontal dashed line denotes the initial plasma density outside of the current sheet - the reference plasma density corresponding to the reference plasma frequency in wavelet spectra.

found that the generation of this electromagnetic emission is stopped when a merging of both the interacting plasmoids into the larger one is finished. That the electromagnetic emission decreases below the initial plasma frequency agrees with the density decrease (see Fig. 6) at this location to the value lower than 50 electrons per cell in the time interval of 160-200 plasma periods. (The initial value, corresponding to the initial plasma frequency, is 60 electrons per cell.) On the other hand, the maximum frequency of electromagnetic pulses corresponds to the plasma frequency inside the plasmoids. The characteristic period of the quasi-periodic electromagnetic emission is 8-10 plasma periods.

We were interested in whether the period of electromagnetic pulses is also expressed in some way by variations in the magnetic field at the interaction location $(x=2400 \Delta, y=300 \Delta)$. The resulting wavelet spectrum of the $B_{x}(x=2400 \Delta)$ component (Fig. 7) confirmed this expectation. Namely, this wavelet spectrum shows enhanced power for variations in the interval 0.09-0.12 ( 0.1) plasma frequency, which corresponds to the estimated quasi-period of $8-10$ plasma periods. No other enhancements in power was recognized in this frequency range. 


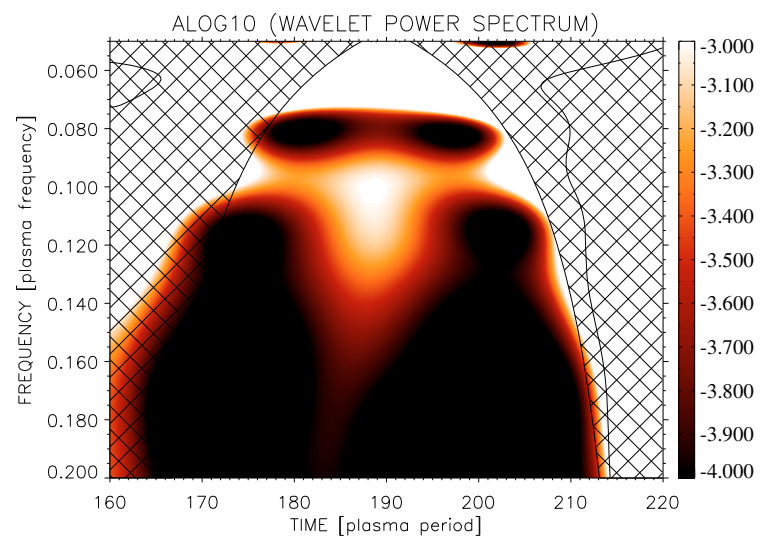

Fig. 7. The wavelet spectrum of the $B_{\mathrm{x}}$ component of the magnetic field at the location $x=2400 \Delta$ and $y=300 \Delta$ at times $160-220$ plasma periods.

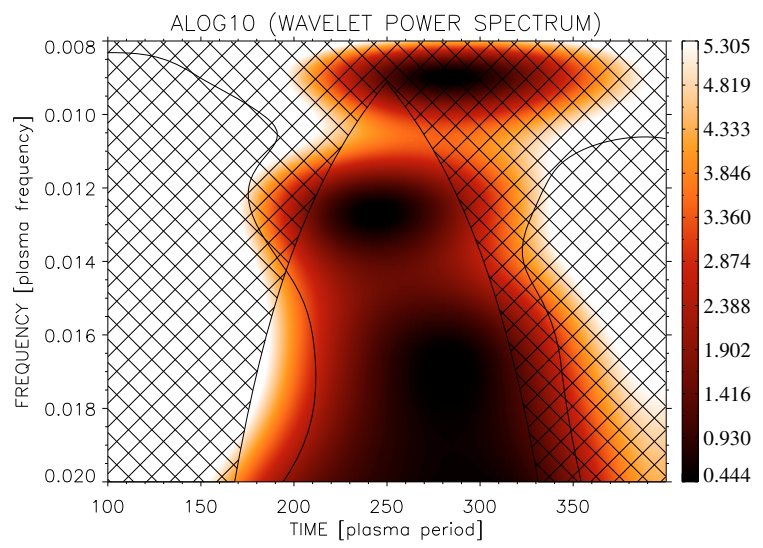

Fig. 8. The wavelet spectrum of the $B_{\mathrm{x}}$ component of the magnetic field averaged over the location interval $x=2400-2600 \Delta$ and $y=300 \Delta$ at times $100-400$ plasma periods.

Furthermore, when studying the wavelet spectra of the $B_{x}$ component averaged over the location interval of $x=$ 2400-2600 $\Delta$ we found a periodic process with the frequency about 0.01 plasma frequency (Fig. 8), i.e. a process with the period of about 100 plasma periods. (The spectra of $B_{y}$ component are similar to those of the $B_{x}$.) We found that this period corresponds to the MHD oscillation of the coalescing plasmoid as described by Tajima et al. (1987). This oscillation can be even seen in Fig. 4 as a wave form of the thick lines delimiting the plasmoids, such as the lines around the plasmoid at $x=2400 \Delta$.

To learn more about details in radio emission processes, we made additional computations of electric and magnetic fields in the vicinity of the interacting plasmoids, i.e. in the subdomain $(x=2200-2600 \Delta, y=300-400 \Delta)$ in the time interval 120-270 plasma periods. Then in this subdomain we separated the electric field in waves into longitudinal (Langmuir - electrostatic) and transversal (electromagnetic) components. Figure 9, which presents the energy densities of both these components at two different instants, shows that the Langmuir waves are accumulated mainly in the interacting plasmoids and that they have a patchy structure. (The maximum energy density of Langmuir waves is $\sim 80$ times greater than for the electromagnetic transverse mode.) On the other hand, packets of the electromagnetic transverse (radio) waves appeared at boundaries of plasmoids and moved outwards and mutually interfered. Some of these electromagnetic wave packets were even reflected at boundaries of dense plasmoids, see the arrow in Fig. 10. a)
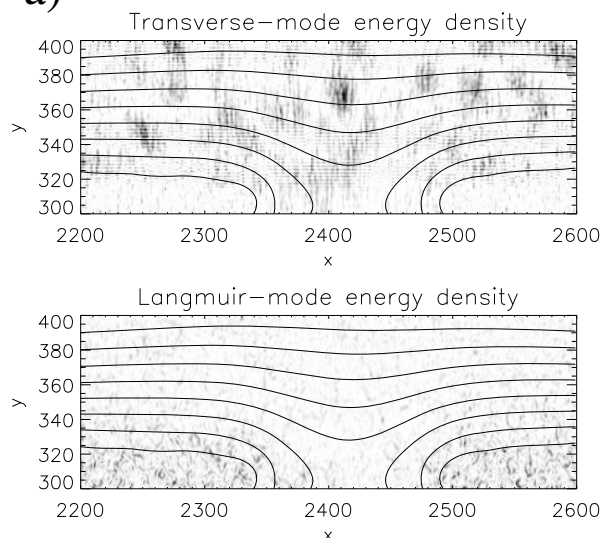

b)
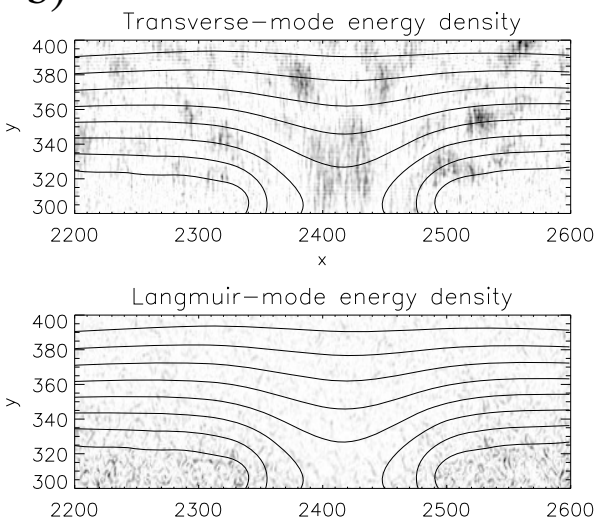

Fig. 9. Transverse and Langmuir mode energy densities at two instants: a) 178 and b) 179 plasma period (black means high-energy densities).

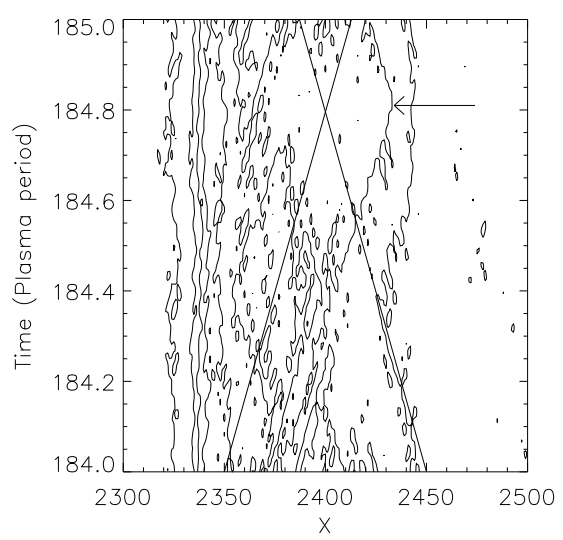

Fig. 10. Detailed view of the contours of the $E_{\mathrm{z}}$ electric field component along the line $y=300 \Delta$ (see Fig. 3 ) in the spatial interval $x=2300-2500 \Delta$ during one plasma period 184-185. For comparison two full lines corresponding to an agent propagation with the speed-oflight are added. The arrow shows the position of reflection of electromagnetic waves.

We analyzed the $E_{z}$ and $B_{x}$ (and $B_{y}$ ) field components in many locations along the $y=300 \Delta$ line. As a second example of the electromagnetic (radio) spectrum, we present what was computed at the location of $x=950 \Delta$ (Fig. 11). As mentioned above, the electromagnetic pulsations end at 600 plasma period, i.e. just at the instant of coalescence for two smaller plasmoids to a larger one; see the joint point of thick lines (the coalescence 
M. Karlický et al.: Radio spectra and reconnection processes

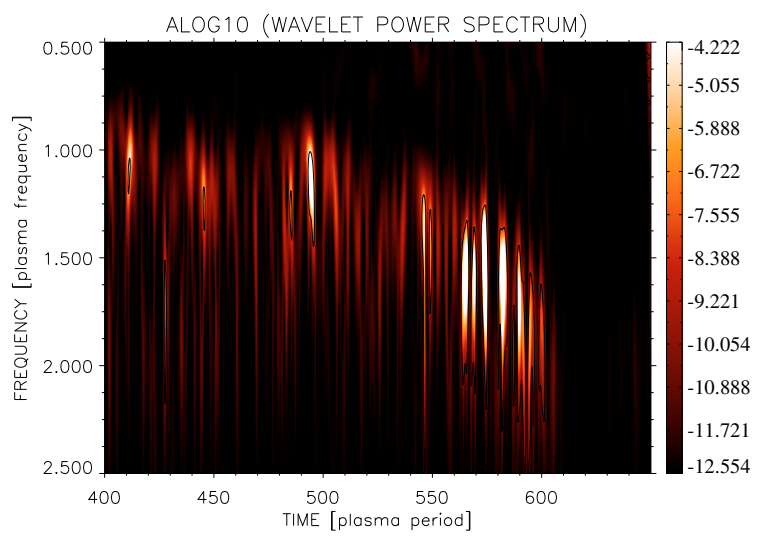

Fig. 11. The wavelet spectrum of the $E_{\mathrm{z}}$ component of the electric field at the location $x=950 \Delta$ and $y=300 \Delta$.

of plasmoids) at the vertical line in Fig. 4, at $x=950 \Delta$, at about 600 plasma periods.

\section{Discussion}

The sizes of computed plasmoids are about 7 orders of magnitude smaller than those that are supposed to produce observed DPSs. This large difference in scales and scale dependent dissipative processes makes impossible to reliably re-scale the results. Nevertheless, there are several physical scales in our simulations. For the coalescence of two plasmoids at $x=2400 \Delta$, we find that (i) the duration of the electromagnetic emission, which corresponds to that of the coalescence process, is 150 plasma periods; (ii) the period of oscillations of the resulting plasmoid is 100 plasma periods; and (iii) the period of electromagnetic pulses is $8-10$ plasma periods. These last two periods were also found in the magnetic field of the plasmoid.

In reality, many plasmoids of different sizes interact simultaneously in their cascade (Bárta et al. 2009). Thus the observed DPS is a result of multi-periodical processes, in agreement with the power-law distribution of the time scales found in DPSs (Karlický et al. 2005).

In Figs. 5 and 11 a global frequency drift of the pulsating structure can be seen similarly as in DPSs. But this drift is not caused by gravitational stratification of the solar atmosphere as in observed DPSs (since no gravity is assumed in our model). This drift is caused by a variation in the plasma density around the plasmoids. Because plasmoid sizes in the present model (hundreds of Debye lengths) are much smaller than those in observed plasmoids (thousands of $\mathrm{km}$ ), the variation in the model gives the frequency drift, which is much faster than observed in DPSs, such that the model drifts cannot be compared with observed ones. On the other hand, the frequency drifts of the DPSs in the gravitationally stratified atmosphere were studied in the magnetohydrodynamic model, see the paper by Bárta et al. (2008a). However, MHD models cannot describe the radio emission processes presented here.

\section{Conclusions}

Both the numerical simulations of the tearing and coalescence processes in the current sheet and the subsequent wavelet analysis of the computed electric field show the spectra that resemble the radio spectra with DPSs. The pulses of electromagnetic emission are generated at a location between two interacting plasmoids just before the coalescence of two plasmoids into a larger one.

We found that the Langmuir waves accumulate in the interacting plasmoids, in agreement with the locations of superthermal electrons (see Drake et al. 2005; and Karlický \& Bárta 2007). On the other hand, the electromagnetic waves appear at the boundaries of the plasmoids and then move outwards, where they mutually interfere and give the short-period pulsations. From observational point of view the most important fact is that the whole interval of electromagnetic emission corresponds to the time of coalescence of two plasmoids.

The electron plasma frequency is the main and dimensionless variable in the present study. Therefore, a broad range of its values can be considered and the model is thus physically meaningful for many astrophysical plasma objects. For example, it is known that some radio spectra of the flare activity on AD Leonis are similar to what is shown in Fig. 1, and see the paper by Osten \& Bastian (2008). This means that the model presented here can also be applicable to these star spectra.

Acknowledgements. The authors thank the editor for very useful comments improving the paper. This research was supported by the grants IAA300030701 (GA AS CR), 205/07/1100 (GA CR), the research project AV0Z10030501 of the Astronomical Institute AS CR, VEGA grant No. 2/0064/09 of the Slovak Academy of Sciences, and the Centre for Theoretical Astrophysics, Prague. The program of mobility between the academies of the Czech Republic and Slovakia is also acknowledged. The wavelet analysis was performed using the software based on tools provided by C. Torrence and G. P. Compo at http://paos. colorado.edu/research/wavelets.

\section{References}

Aschwanden, M. J. 2004, Physics of the Solar Corona (Germany, Berlin: Springer-Verlag), 17

Bárta, M., Karlický, M., \& Žemlička, R. 2008a, Sol. Phys., 253, 173

Bárta, M., Vršnak, B., \& Karlický, M. 2008b, A\&A, 477, 649

Bárta, M., Büchner, J., \& Karlický, M. 2010, Adv. Space Res., 45, 10

Drake, J. F., Shay, M. A., Thongthai, W., \& Swisdak, M. 2005, Phys. Rev. Lett., 94 (9), 095001

Jiřička, K., Karlický, M., Kepka, O., \& Tlamicha, A. 1993, Sol. Phys., 147, 203 Karlický, M. 2004, A\&A, 417, 325

Karlický, M., \& Bárta, M. 2007, A\&A, 464, 735

Karlický, M., Bárta, M., Mészarosová, H., \& Zlobec, P. 2005, A\&A, 432, 705

Khan, M., Vilmer, N., Saint-Hilaire, P., \& Benz, A. O. 2002, A\&A, 388, 363

Kliem, B., Karlický, M., \& Benz, A. O. 2000, A\&A 360, 715

Tajima, T., Sakai, J., Nakajima, H., et al. 1987, ApJ, 321, 1031

Ohyama, M., \& Shibata, K. 1998, ApJ, 499, 934

Osten, R. A., \& Bastian, T. S. 2008, ApJ, 674, 1078

Torrence, C., \& Compo, G. P. 1998, Bull. Am. Meteorol. Soc., 79, 61

Saito, S., \& Sakai, J. I. 2004, ApJ, 616, L179 